Trauma Berufskrankh 2016 • [Suppl 1]: 18:S73-S77 DOI 10.1007/s10039-015-0040-6

Online publiziert: 6 . Juni 2015

(c) Springer-Verlag Berlin Heidelberg 2015

U.-J. Gerlach · C. Grimme · R. Schoop · M. Borree

Abteilung für Septische Unfallchirurgie und Orthopädie,

Berufsgenossenschaftliches Unfallkrankenhaus Hamburg, Hamburg, Deutschland

\title{
Behandlungsstrategien bei Gelenkinfektionen
}

$[1,9,12,14])$ oder nach Arthrotomien (Inzidenz $\leq 1 \%$; $[1,10,18]$ ). Seltener sind Gelenkinfektionen bedingt durch paraartikulär fortgeleitete Infekten, wie z. B. eine verschleppte Bursitis.

Nach Eintritt eines bakteriellen Erregers in den Kniebinnenraum kommt es zu einer intraartikulären Erregervermehrung und zur Anreicherung von Stoffwechselprodukten bzw. Toxin. $\mathrm{Zu}$ beobachten ist nach $24 \mathrm{~h}$ die Freisetzung lysosomaler Enzyme, nach $48 \mathrm{~h}$ kommt es zum klinischen Infektbeginn. Im Verlauf kommt es zur Freisetzung von Glycosaminen und Leukozytenproteinasen [13], nach 5 Tagen Beginn der Knorpelzerstörung, die nach 10 Tagen irreversibel ist. Bereits nach 3 Tagen kommt es zu einer Synoviahypertrophie, nach 11 Tagen zur Pannusbildung. Am 17. Tag nach Infektbeginn ist ein Kapseldurchbruch festzustellen. Untersuchungen haben gezeigt, dass die Arthroserate einen deutlichen Anstieg bereits am 3. Tag nach Beginn der Gelenkinfektion zeigt.

\section{Diagnosestellung}

Die Diagnosestellung eines akuten Gelenkinfektes ist oft einfacher als die Diagnosestellung eines chronischen Gelenk- infektes. Die bekannten klinischen Entzündungszeichen (Rubor/Calor/Dolor/ Functio laesa; - Abb. 1) sind bei der akuten Gelenkinfektion häufig eindrucksvoll ausgeprägt.

Die schmerzhaft aufgehobene Beweglichkeit des betreffenden Gelenkes verbunden mit erhöhten Temperaturen ist typisch für die akute Gelenkinfektion. Bei chronischer, lange Zeit schon bestehender Gelenkinfektion können die klinischen Entzündungszeichen fehlen oder sind nur deutlich abgeschwächt vorhanden (• Abb. 2).

Für die Diagnostik wichtig ist eine genaue Anamneseerhebung mit Befragung nach vorausgegangenen, auch längere Zeit zurückliegenden Gelenkpunktionen oder intraartikulären Injektionen oder Arthroskopien. Selbstverständlich muss auch nach Grunderkrankung wie u. a. Diabetes mellitus, primärer chronischer Polyarthritis (PCP), möglichen gelenkfernen Streuherden (Tonsillitis, Otitis, Harnwegsinfekt) oder immunsuppressiven Medikamenten gefragt werden.

Die Laborparameter (Leukozyten/ CRP/BSG; [19]) zeigen nur bei Frühinfekt eine deutliche Erhöhung. Bei chronischen Gelenkinfektionen sind diese genannten

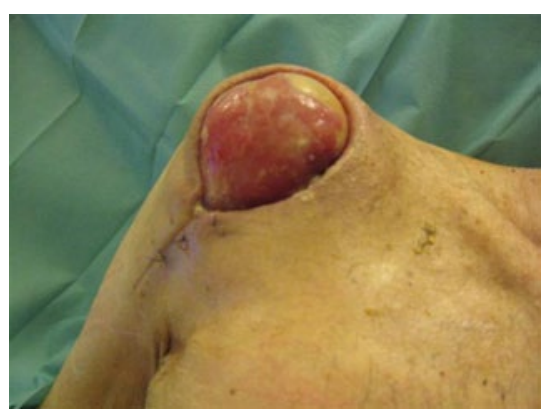

Abb. $2 \Delta$ Klinisches Bild eines chronischen

Schultergelenkempyems
Abb. $1<$ Klinisches Bild einer Fibulaosteitis mit akutem Empyem des oberen Sprungge-

lenkes

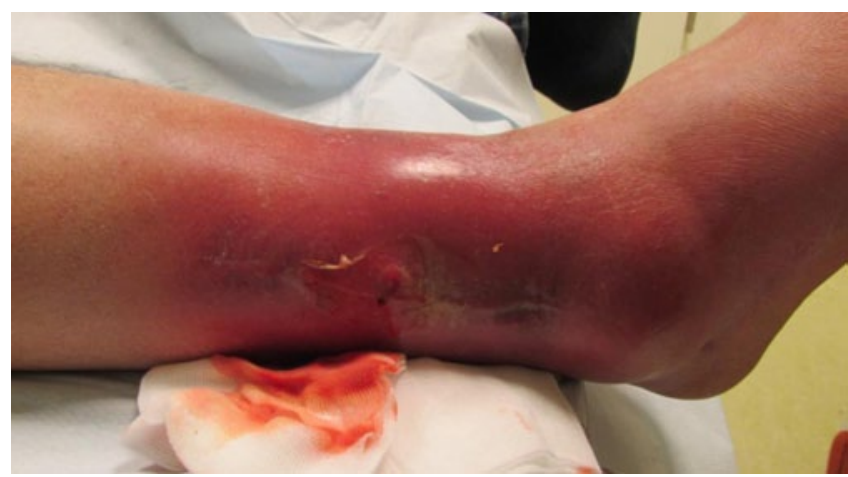



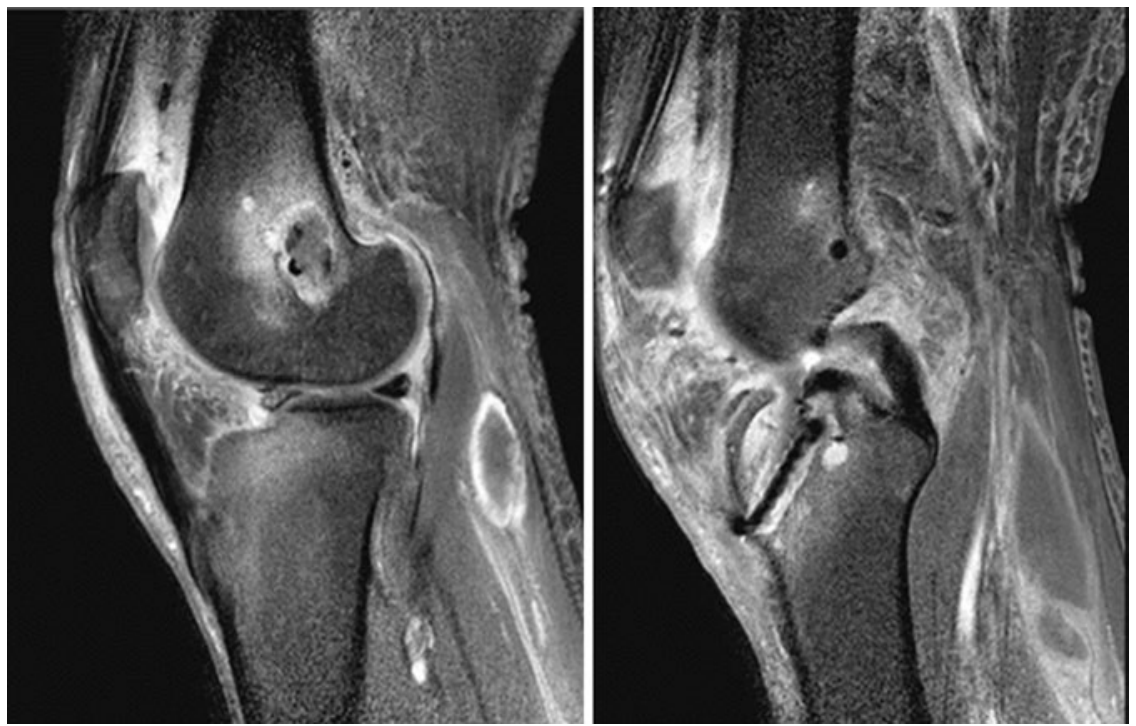

Abb. 3 A Magnetresonanztomographie bei chronischem Kniegelenkempyem mit Infekt des tibialen und femoralen Bohrkanals sowie Abszedierung in der Wade

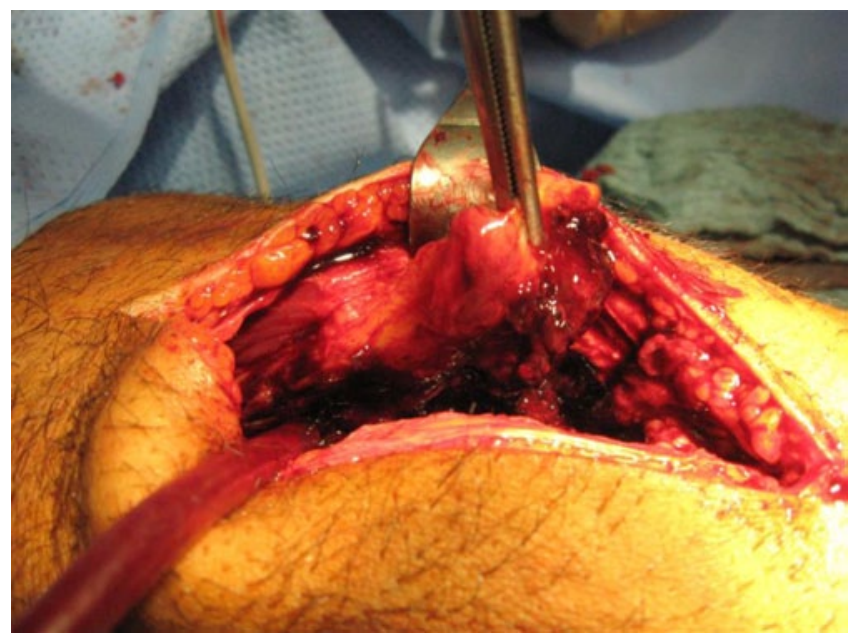

Abb. $4<$ Hypertrophe Synovia bei chronischem Kniegelenkempyem

Laborparameter wenig aussagekräftig, haben allenfalls hinweisenden Charakter.

Weiterer wichtiger Baustein in der Diagnostik der Gelenkinfektion ist die Gelenkpunktion. Die Inspektion des aspirierten Punktats lässt differenzialdiagnostische Überlegungen zu. Das Punktat kann serös, serös-flockig, trübe bis eitrig sein. Ein Teil des Gelenkpunktats sollte zur bakteriologischen Untersuchung in ein mikrobiologisches Labor verschickt werden.

Ein weiterer Teil des Gelenkpunktats sollte unter Gram-Färbung im Mikroskop untersucht werden. $\mathrm{Zu}$ überprüfen ist die Leukozytenzahl im Punktat, ggf. auch ein Keimnachweis. Bei Leukozyten im Punktat $\geq 25.000 / \mu \mathrm{l}$ [5] ist von einem Empyem auszugehen. Sollte im Punktat der Direkt- nachweis eines Erregers gelingen, so wäre die Diagnose der Gelenkinfektion gestellt [23]. Als neues Diagnostikum steht der Nachweis von $\alpha$-Defensin zur Verfügung. Bei $\alpha$-Defensin handelt es sich um ein antimikrobielles Peptid, dass bei Eindringen eines Pathogens in die Synovialis sezerniert wird. Hier bindet es sich an die Zellmembran des Bakteriums und führt zum Absterben des Keimes [7]. Die Bestimmung von $\alpha$-Defensin ist in der Diagnostik einer periprothetischen Infektion ein geeigneter Parameter, aber auch in der Diagnostik der Gelenkinfektion ohne Prothese. Die Spezifität und Sensitivität des Tests liegt bei 95-97\% [8].

Als bildgebende Verfahren zur Diagnostik eines Kniegelenkinfektes sind die Röntgennativuntersuchung, die Sonogra- phie und die Magnetresonanztomographie zu nennen. Die konventionelle Röntgenaufnahme zeigt im Frühinfekt keinen Hinweis auf entzündliche Veränderungen mit Ausnahme von Lufteinschlüssen bei perforierenden Verletzungen oder Infektion mit gasbildenden Keimen. Bei der chronischen Gelenkinfektion bzw. dem Spätinfekt zeigen sich Veränderungen wie Sequestrierung und Aufhellungen unterhalb des Knorpels oder auch eine Zerstörung der Gelenkflächen [4]. Magnetresonanztomographisch zeigen sich meist ein Ödem von angrenzendem Knochen und Knorpel, gelegentliche Arrosionen am Knochen und Knorpel und der meist bereits klinisch bekannte Erguss [4, 17]. Die Synoviahypertrophie ist im der Magnetresonanztomographie (MRT) gut zu diagnostizieren ( $\bullet$ Abb. 3). Die Sonographie $[4,16,17]$ kann den klinisch schon bekannten Gelenkerguss nachweisen, aber auch eine Synoviaverdickung lässt sich sonographisch feststellen.

Die Computertomographie (CT)Untersuchung sowie die Dreiphasenskelettszintigraphie haben in der Diagnostik einer Gelenkinfektion eine nachgeordnete Bedeutung $[4,17]$.

Zusammenfassend ist zu sagen, dass für die Diagnosestellung einer Gelenkinfektion die Anamnese, die klinischen Symptome, Laboruntersuchungen, die Gelenkpunktion und Sonographie bzw. MRT entscheidend sind. Die Nativröntgenuntersuchung ist zur Diagnose einer akuten Gelenkinfektion nicht geeignet.

\section{Therapie}

Ziel der Therapie von Gelenkinfektionen sind die dauerhafte Infektberuhigung und der Erhalt eines stabilen Gelenkes sowie der Erhalt einer belastungsfähigen Extremität. Die Mobilität des Patienten soll möglichst wiederhergestellt werden, die berufliche und soziale Wiedereingliederung sollen ermöglicht werden.

$\mathrm{Zu}$ unterscheiden in der Therapie ist die akute von der chronischen Infektion. Aufgrund der oben genannten pathophysiologischen Veränderungen ist von einer akuten Infektion innerhalb der ersten 7 Tage nach erstmaliger Manifestation der klinischen Symptome zu spre- 
chen, darüber hinaus ist von einer chronischen Gelenkinfektion auszugehen [13].

Die Behandlung von akuten Gelenkinfektionen stellt einen unfallchirurgischen Notfall dar! Die akute Gelenkinfektion bedarf der sofortigen operativen Intervention durch einen darin erfahrenen Operateur.

Die Diagnosestellung einer Gelenkinfektion ist zu erzwingen, die Behandlung hat zeitnah, radikal und mit Konzept zu erfolgen. Verzögerungen in der Diagnostik oder eine insuffiziente Therapie führen zu einer ausbleibenden Infektberuhigung mit desaströsen Folgen für den $\mathrm{Pa}$ tienten. Die Aufarbeitung von 90 Patienten, die mit Schultergelenkempyem bei uns behandelt wurden, ergab vor Übernahme eine Dauer der Behandlung von durchschnittlich 123 Tagen mit $\varnothing 5,5$ (1->20) ,infektberuhigenden Voroperationen " (ohne Primäroperation). Ähnliche Ergebnisse erbrachte die Analyse von 84 Patienten mit chronischem Kniegelenkempyem. Die durchschnittliche Dauer der Vorbehandlung vor Aufnahme bei uns betrug 90 Tage (11 bis 600 Tage) mit $\varnothing$ 4,5 (0-12) Voroperationen.

Für den Behandlungserfolg ist das radikale chirurgische Vorgehen entscheidend. Die richtige Behandlung ist nicht nur davon abhängig, ob eine akute oder chronische Infektion vorliegt, sondern auch von der Vorbehandlung und Infektausdehnung sowie dem Ausmaß der Gelenkschädigung.

Bei der stadienadaptierten Behandlung $[11,23,24]$ steht das arthroskopische oder das offene Behandlungsverfahren zur Auswahl. Akute Empyeme werden arthroskopisch behandelt, chronische Empyeme in der Regel offen chirurgisch. Bereits offen vorbehandelte Empyeme werden offen weiter behandelt, Gelenkinfektionen mit Infektion des gelenknahen Knochens können nicht arthroskopisch behandelt werden, auch hier ist eine offene Behandlung notwendig.

Die arthroskopische Behandlung ist sinnvoll und indiziert bei akutem Empyem und Empyemen Stadium I und II nach Gächter ([11]; I: Synovialishyperämie und -erguss, II: Synovialishypertrophie, Eiteransammlung im Gelenk). Die Arthrotomie zur Behandlung einer Gelenkinfektion ist notwendig bei chronischem Em-

Trauma Berufskrankh 2016 • [Suppl 1]: 18:S73-S77 DOI 10.1007/s10039-015-0040-6

(c) Springer-Verlag Berlin Heidelberg 2015

\section{U.-J. Gerlach · C. Grimme · R. Schoop · M. Borree}

\section{Behandlungsstrategien bei Gelenkinfektionen}

\section{Zusammenfassung}

Hintergrund. Die Gelenkinfektion stellt eine schwerwiegende Komplikation dar und führt bei ausbleibender oder inadäquater Behandlung zur irreversiblen Gelenkzerstörung. Ursächlich für eine Gelenkinfektion sind endogen-hämatogene und exogene Faktoren. In unserem Patientengut sind die Empyeme fast ausschließlich verursacht durch iatrogene Maßnahmen (arthroskopische Operationen, Punktionen, intraartikuläre Infektionen), oder sie sind Folge gelenknaher Frakturen und penetrierender Verletzungen.

Diagnostik. Zur Diagnosestellung notwendig ist neben Laboruntersuchung, NativRöntgen- und MRT-Untersuchung die Gelenkpunktion mit Direktabstrich. Die Leukozytenzahl ist zu bestimmen (Grenzwert: 25.000/ $\mu \mathrm{l})$, und in der Gram-Verfärbung sollte mikroskopisch die Identifizierung möglicherweise vorliegender Bakterien erfolgen.

Therapie. Bei dem akuten Gelenkempyem

handelt es sich um einen unfallchirurgischen
Notfall, der sofort operiert werden muss, da aufgrund der pathophysiologischen Vorgänge rasch irreversible Knorpelschäden auftreten. Das akute Empyem ist arthroskopisch zu behandeln. Von einem chronischen Empyem ist auszugehen, wenn die klinischen Symptome länger als 7 Tage bestehen. Die Behandlung des chronischen Empyems erfolgt durch Arthrotomie, Synovialektomie und Entfernung des einliegenden Fremdmaterials inklusive von Kreuzbandersatz. Postoperativ ist von Beginn an eine intensive Übungsbehandlung notwendig, auch unter Einsatz von Schmerzkathetern. Die Verlegung in ein Zentrum für septische Chirurgie ist sinnvoll.

\section{Schlüsselwörter}

Komplikation - Empyem - Synovialektomie . Notfall $\cdot$ Arthrotomie

\section{Treatment strategies for joint infections}

\section{Abstract}

Background. Joint infections represent a severe complication that results in irreversible joint destruction when inadequately treated. The causes of joint infections consist of endogenous, hematological and exogenous factors. The patients in this cohort acquired empyema almost solely through iatrogenic means (e.g. arthroscopic operations, punctures and intra-articular infections) or as a result of fractures close to joints and penetrating injuries.

Diagnostics. In order to make a correct diagnosis, a joint puncture with a direct swab test is necessary in addition to laboratory examinations, $x$-ray and magnetic resonance imaging (MRI). The amount of leucocytes is to be determined (threshold value $25,000 / \mu \mathrm{l}$ ) as well as gram staining to identify any bacteria present.

Therapy. Acute joint infections are considered to be orthopedic emergencies and im- mediate surgery is necessary, as irreversible cartilage damage can quickly result due to pathophysiological processes. Acute joint empyema must be treated arthroscopically. Clinical symptoms lasting more than 7 days result in chronic empyema. Chronic empyema should be treated by arthrotomy, synovectomy and the removal of extraneous material including cruciate ligament replacement. Postoperatively, an immediate intensive physiotherapy is necessary, even under the use of pain catheters and transfer to a center specialized in septic surgery is warranted.

Keywords

Complication · Empyema · Synovectomy . Emergency $\cdot$ Arthrotomy pyem und bei Empyem Stadium III und IV nach Gächter (III: Synovialisschwamm und beginnender Knorpelschaden, IV: "Synovialismalignität").

Die arthroskopische Behandlung bedarf einer sorgfältigen Inspektion des Gelenkes. Ein radikales Débridement $[11,15$,
24] ist erforderlich, ebenso die Lavage mit mindestens 101 Flüssigkeit und die Einlage von Antibiotikumträgern. Diese antibiotikumhaltigen Vliese können über den Arbeitstrokar in das Gelenk eingebracht werden. Bei Infektion nach Vorderkreuzbandersatzplastik sollte ein Erhaltungs- 

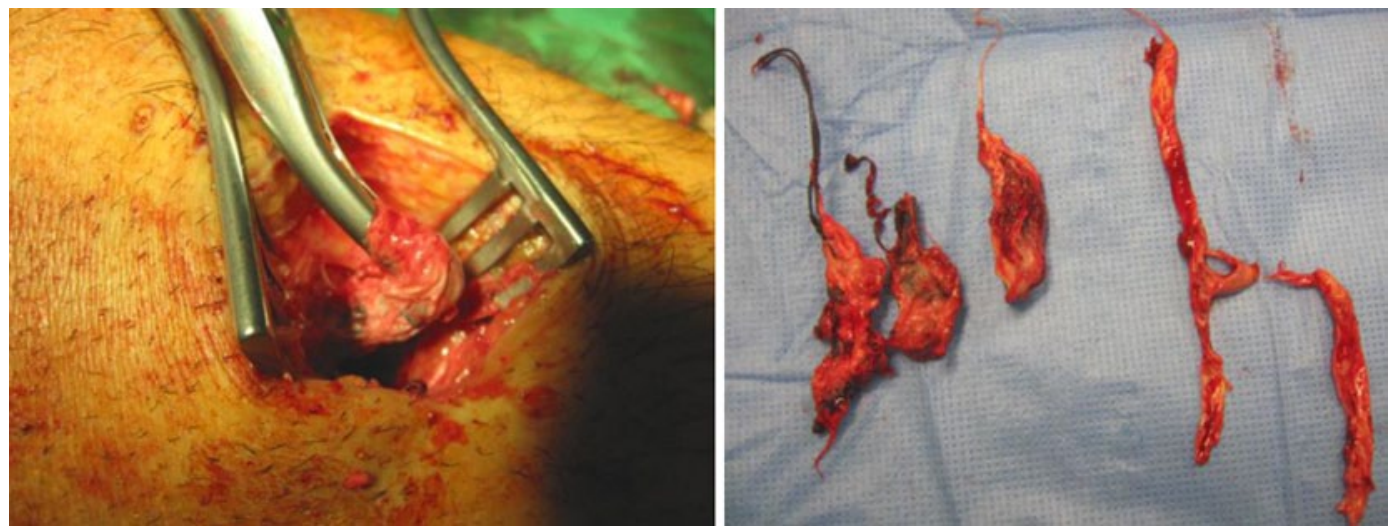

Abb. $5<$ Entfernter infizierter Ersatz des vorderen Kreuzbandes

versuch des Kreuzbandersatzes vorgenommen werden [3]. Bei der Behandlung von akuten Empyemen ist eine geplante Second-look-Operation nach 48$72 \mathrm{~h}$ sinnvoll.

Bei fortgeschrittenen Gelenkinfektionen sollte, wenn die Behandlung arthroskopisch begonnen wurde, frühzeitig der Umstieg auf ein offenes Verfahren durchgeführt werden. Die Gefahr der Arthroskopie liegt darin, dass häufig das Problem unterschätzt wird. Es resultiert eine nicht ausreichend radikale Behandlung der Gelenkinfektion.

Beim offenen chirurgischen Vorgehen sollte die bilaterale Arthrotomie durchgeführt werden (Kniegelenk, Ellenbogengelenk, oberes Sprunggelenk). Am Schultergelenk ist zumindest bei chronischen Infektionen neben dem ventralen Zugang der dorsale Zugang erforderlich, um radikal débridieren zu können. Ein Hüftgelenkempyem sollte stets offen chirurgisch über einen Zugang behandelt werden, nicht arthroskopisch.

Bei der offenen chirurgischen Behandlung ist die radikale Synovialektomie (• Abb. 4) notwendig. Begleitende Infektionen von gelenknahem Knochen müssen durch radikale Sequestrektomie in gleicher Sitzung mit behandelt werden [20, 25]. Implantate und Kreuzbandersatz müssen entfernt werden (• Abb. 5). Nach radikalem Débridement erfolgt die Einlage lokaler resorbierbarer Antibiotikumträger. Das Gelenk wird verschlossen. Selbstverständlich wird entnommenes Gewebematerial zur bakteriologischen und histologischen Untersuchung verschickt. Eine kurzfristige systemische Antibiose (7 bis 10 Tage) ist postoperativ erforderlich.
Die postoperative Physiotherapie ist entscheidend und unerlässlich [23]. Zunächst unter ausreichender Schmerzmedikation ggf. auch unter Schmerzkatheterbehandlung erfolgt die Umlagerung auf einer Kirschner-Schiene, später die Behandlung mit der Motorschiene und die aktive Übungsbehandlung. Parallel zu dem operativen Vorgehen ist ein intensives Rehabilitationsprogramm unter krankengymnastischer Anleitung, hydrophysikalischer, sporttherapeutischer und ergotherapeutischer Behandlung erforderlich. Hilfsmittelversorgung und ggf. Einleitung berufshelferischer Maßnahmen können im Verlauf notwendig werden. Die begonnene Physiotherapie sollte unbedingt auch nach Entlassung aus der stationären Behandlung fortgeführt werden.

\section{Fazit für die Praxis}

- Bei Verdacht auf Gelenkinfektionen ist eine Diagnose zu erzwingen.

- Eine akute Gelenkinfektion stellt einen unfallchirurgischen Notfall dar.

- Die Behandlung hat stadienadaptiert zu erfolgen, entweder arthroskopisch oder offen chirurgisch.

- Für den Behandlungserfolg ist das radikale chirurgische Vorgehen entscheidend.

- Bei fortgeschrittenen Gelenkinfektionen sollte, wenn arthroskopisch begonnen wurde, frühzeitig der Umstieg auf ein offenes Verfahren durchgeführt werden. Die Gefahr der Arthroskopie liegt darin, dass häufig das Problem unterschätzt wird.

- Bei chronischem oder voroperiertem Empyem ist das offene Vorgehen er- forderlich, hier ist ein arthroskopisches Vorgehen nicht sinnvoll.

- Die Synovektomie hat radikal zu erfolgen.

- Entscheidend für die Infektberuhigung ist das radikale operative Vorgehen.

- Die Einlage lokaler Antibiotikumträger und die kurzzeitige systemische Antibiose sind unterstützend und entbinden nicht von einer radikalen chirurgischen Behandlung.

- Nach operativer Behandlung ist der sofortige Beginn mit intensiver Übungsbehandlung unerlässlich für das funktionelle Ergebnis.

- Bei weitgehender Gelenkzerstörung und oder persistierender Instabilität ist die Arthrodese indiziert.

- Gelenkinfektionen werden nicht selten zu spät erkannt und nicht suffizient chirurgisch behandelt. Konsequenz hieraus sind lange Krankheitsverläufe mit vielen frustranen Voroperationen und ein schlechteres funktionelles Ergebnis. Das Heilverfahren und der Zeitraum der Arbeitsunfähigkeit dauern länger an, es resultiert in der Regel eine höhere Minderung der Erwerbsfähigkeit (MdE). Sinnvoll ist deswegen die frühzeitige Verlegung in ein septisches Zentrum.

\section{Korrespondenzadresse}

\section{Dr. U.-J. Gerlach}

Abteilung für Septische Unfallchirurgie und Orthopädie

Berufsgenossenschaftliches Unfallkrankenhaus Hamburg

Bergedorfer Str. 10, 21033 Hamburg

U.J.Gerlach@buk-hamburg.de 


\section{Einhaltung ethischer Richtlinien}

Interessenkonflikt. U.-J. Gerlach, C. Grimme, R. Schoop und M. Borree geben an, dass kein Interessenkonflikt besteht.

Dieser Beitrag beinhaltet keine Studien an Menschen oder Tieren.

The supplement containing this article ist not sponsored by industry.

\section{Literatur}

1. Anders G (1984) Gelenkpunktionen und intraartikuläre Injektionen in ambulanten orthopädischen Einrichtungen. Beitr Orthop Traumatol 31:419-425

2. Armstrong RW, Bolding F, Joseph R (1992) Septic arthritis following arthroscopy: clinical syndromes and analysis of risk factors. Arthroscopy 8:213-223

3. Attmanspacher W, Dittrich V, Stedtfeld W (1997) Behandlungsstrategie beim Kniegelenkinfekt nach vorderer Kreuzbandplastik. Arthroskopie 10:318322

4. Braunschweig $\mathrm{R}$, Bergert $\mathrm{H}$, Kluge $\mathrm{R}$, Tiemann $\mathrm{AH}$ (2011) Bildgebende Diagnostik bei Osteitis/Osteomyelitis und Gelenkinfekten. Z Orthop Unfall 149:436-448

5. Chapman MW (2001) Chapman's orthopaedics, 3. Aufl. Lippincot, Philadelphia, S 1800

6. D'Angelo GL, Ogilvie-Harris DJ (1988) Septic arthritis following arthroscopy, with cost/benefit analysis of antibiotic prophylaxis. Arthroscopy 4:10-14

7. Deirmengian C, Kardos K, Kilmartin P, Cameron A, Schiller K, Booth R, Parvizi (2014) Combined measurement of synovial fluid a-Defensin and C-reactive protein levels: highly accurate for diagnosing periprosthetic joint infection. J Bone Joint Surg Am 96:1439-1445

8. Deirmengian C, Kardos K, Kilmartin P, Cameron A, Schiller K, Booth R, Parvizi (2015) The Alpha-defensin Test for periprosthetic joint infection outperforms the Leokocyte Esterase Test Strip. Clin Orthop Relat Res 473:198-203

9. Diefenbeck M, Abitzsch D, Hofmann GO (2012) Die Gelenkinfektion. Bekanntes und neue Trends. Unfallchirurg 115:489-495

10. Dittrich V, Attmanspacher W, Stedtfeld HW (2002) Gelenkinfektionen. Chirurg 73:959-967

11. Gächter A (1994) Gelenkinfekt: Arthroskopische Spülbehandlung - Hints und Tricks. Arthroskopie 7:98-101

12. Gray RG, Gottlieb NL (1983) Intra-articular corticosteroids. An updated assessment. Clin Orthop Relat Res 177:235-263

13. Heppert V, Glatzel U, Wagner CH, Wentzensen A (2002) Komplikationsmanagement bei infizierten Kniegelenken. Trauma Berufskrankh 4:63-71

14. Holland Ch, Jaeger L, Smentkowski U, Weber B, Otto $\mathrm{CH}$ (2012) Septische und aseptische Komplikationen in Verbindung mit Kortikoid-Injektionen: Eine Auswertung von 278 einschlägigen Fällen der Gutachterkommissionen und Schlichtungsstellen der Jahre 2005-2009. Dtsch Arztebl Int 109(24):425-430. doi:10.3238/arztebl.2012.0425

15. Jerosch J (2004) Akuter Gelenkinfekt. Orthopade 33:1309-1320

16. Jerosch J, Prymka M (1998) Arthroskopische Therapie der septischen Arthritis - Operative Technik und Ergebnisse. Unfallchirurg 101:454-460

17. Linke R, Weidemann H, Militz M (2009) Bildgebende Diagnostik der Osteitis. Szintigraphie, PET oder MRT? Trauma Berufskrankh 11(Suppl 2):193-202
18. Pioro MH, Mandell PF (1997) Septic Arthritis. Rheum Dis Clin North Am 23:239-258

19. Riel KA, Primbs J, Bernett P (1994) Arthroskopische Überlaufsspüldrainage bei akuter postoperativer Kniegelenksinfektion - Langzeitergebnisse. Chirurg 65:1023-1027

20. Schmidt HGK, Gerlach UJ, Hadler D, Wurm M (2003) Therapie des posttraumatischen Ellengelenkempyems. Trauma Berufskrankh 5:55-64

21. Simank HG, Wadi B, Bernd L (2004) Gelenkempyeme. Orthopäde 33:327-331

22. Smith NC (1987) Complications in arthroscopic surgery performed by experienced arthroscopists. Arthroscopy 4(3):215-221

23. Stutz G, Gächter A (2001) Diagnostik und stadiengerechte Therapie von Gelenkinfekten. Unfallchirurg 104:682-686

24. Stutz G, Kuster MS, Kleinstuck F, Gächter A (2000) Arthroscopic management of septic arthritis: stages of infections and results. Knee Surg Sports Traumatol Arthrosc 8:270-274

25. Walenkamp GH, Kleijn LL, Leeuw M de (1998) Osteomyelitis treated with gentamicin - PMMA beads. Acta Orthop Scand 69:518-522 